\title{
Caractérisation hydrogéochimique des eaux souterraines du bassin versant de la Baya, Est Côte d'Ivoire
}

\author{
Germain Kobenan N'GUETTIA ${ }^{1}$, Jules Mangoua Oi MANGOUA², \\ Narcisse Kouassi ABOUA ${ }^{1}$, Aristide Gountôh DOUAGUI ${ }^{1}$ et Lanciné Droh GONE ${ }^{{ }^{*}}$ \\ ${ }^{1}$ UFR-Sciences et Gestion de l'Environnement, Laboratoire Géosciences et Environnement, Université \\ Nangui-Abrogoua, 02 BP 801 Abidjan 02, Côte d'Ivoire. \\ ${ }^{2}$ UFR Environnement, Université Jean Lorougnon Guedé, Daloa, BP 150 Daloa, Côte d'Ivoire. \\ *Auteur correspondant, E-mail :dialou25_9@yahoo.fr; Tel. : +22505043337
}

\section{RESUME}

Les eaux souterraines sont la principale source d'approvisionnement en eau des populations dans le bassin versant de la Baya. Toutefois, les activités anthropiques sur ce Bassin pourraient dégrader la qualité chimique de cette ressource. Afin d'évaluer la qualité physicochimique de ces eaux et déterminer les phénomènes à l'origine de leur minéralisation, des analyses physico-chimiques et traitements statistiques des données hydrochimiques ont été effectuées sur 13 forages et 12 puits. Il ressort que les eaux souterraines du bassin de la Baya sont dans l'ensemble acides avec un $\mathrm{pH}$ moyen de 5,98 et faiblement minéralisées, à l'exception de celles des localités d'Apkokro $\left(1236 \mu \mathrm{S} . \mathrm{cm}^{-1}\right)$ et Tiédo $\left(1092 \mu \mathrm{S} . \mathrm{cm}^{-1}\right)$. Les eaux sont marquées par des faciès chloruré-sulfaté calcique et hydrogénocarbonaté calcique et magnésien. Les teneurs en nitrate, chlorure, silice, manganèse et fer sont parfois élevées et dépassent les valeurs de la directive OMS de potabilité des eaux de consommation. L'Analyse en Composantes Principales (ACP) a mis en évidence deux phénomènes dans l'acquisition du chimisme des eaux : la dissolution de la roche encaissante au contact des eaux et une minéralisation liée à la pollution par apports superficiels des eaux d'infiltration et de lessivage des sols. Dans l'ensemble, la qualité des eaux souterraines est bonne mais nécessite dans certains cas, un traitement spécifique avant consommation.

(C) 2019 International Formulae Group. All rights reserved.

Mots clés: Qualité eau souterraine, minéralisation, pollution, bassin versant de la Baya.

\section{Hydrogeochemical characterisation of groundwater in the Baya watershed, East Côte d'Ivoire}

\begin{abstract}
Groundwater is the main source of water for people in the Baya watershed. However, human activities on this Basin could degrade the chemical quality of this resource. In order to evaluate the physicochemical quality of these waters and determine the phenomena at the origin of their mineralization, physicochemical analyzes and statistical treatments of the hydrochemical data were carried out on 13 boreholes and 12 wells. It appears that the groundwater in the Baya Basin is generally acidic with an average $\mathrm{pH}$ of 5.98 and weakly mineralized, with the exception of the localities of Apkokro $\left(1236 \mu \mathrm{S} . \mathrm{cm}^{-1}\right)$ and Tiédo $\left(1092 \mu \mathrm{S} . \mathrm{cm}^{-1}\right)$. The waters are marked by chlorinated-sulphated calcium and calcium carbonate and magnesium carbonate facies. The levels of nitrates, chlorides, silicas, manganese and irons are sometimes high and exceed the values of the WHO directive drinking water. The Principal Component Analysis (ACP) revealed two phenomena in the acquisition of water chemistry: the dissolution of the host rock in contact with the water and mineralization
\end{abstract}


linked to pollution by superficial inputs of seepage water and soil leaching. Overall, the quality of the groundwater is good but in some cases requires specific treatment before consumption.

(C) 2019 International Formulae Group. All rights reserved.

Keywords: Groundwater quality, mineralization, pollution, Baya watershed.

\section{INTRODUCTION}

Les eaux souterraines constituent une ressource vitale lorsqu'il s'agit de faire face aux besoins en eau douce des collectivités. L'utilisation de ces eaux pour les besoins domestiques s'accroît, certes à cause de la poussée démographique, mais aussi à cause de la pollution et du tarissement des eaux de surface. Tous ces facteurs concourent à la raréfaction de cette ressource naturelle dont la demande se fait toujours plus pressante.

En Côte d'Ivoire, comme partout ailleurs en Afrique, les eaux souterraines sont confrontées à un phénomène de pollution d'origine anthropique qui dégrade leur qualité. Les études réalisées par Blé et al. (2016) et Aké et al. (2012), ont fait cas de cette pollution dans les régions d'Abidjan, d'Agboville et de Bonoua situées au Sud de la Côte d'Ivoire. Il ressort de ces travaux que la pollution des nappes fréquemment ressentie au niveau des centres urbains touche aussi les zones rurales de grande ou petite taille. Pour ces auteurs, la dégradation des ressources en eau souterraine est intimement liée aux activités agricoles et à la décomposition de la litière forestière. Selon l'OMS (2011), la mauvaise qualité de l'eau pose un problème de santé public et cause de nombreuses maladies telles que la dysenterie, la fièvre typhoïde, etc. Il devient donc important de connaître leur qualité pour une utilisation sans risques sanitaires.

Dans le bassin versant de la Baya situé à l'Est de la Côte d'Ivoire, les difficultés liés à la gestion des eaux souterraines alimentant les différentes localités du bassin, constituent un problème majeur auquel ces localités doivent faire face. En effet, pendant la saison sèche, bon nombre de puits et forages tarissent ou sont abandonnés à cause de leur apparence rougeâtre et goût désagréable. En milieu urbain comme rural, ces ouvrages sont sous l'influence des effluents domestiques et des engrais agricoles. Par ailleurs, la surexploitation de ces ressources en vue de couvrir les besoins quotidiens en eau, détériore davantage leur qualité. Plusieurs études ont déjà été réalisées dans le bassin de la Baya, notamment sur leur vulnérabilité à la pollution (Mangoua, 2013). Celles portant sur la qualité physico-chimique sont sommaires et les mécanismes qui gouvernent le chimisme de ces eaux restent encore très peu connus.

L'objectif de ce travail est d'évaluer la qualité physico-chimique et de déterminer les processus à l'origine de la minéralisation des eaux souterraines du bassin versant de la Baya.

\section{MATERIEL ET METHODES \\ Présentation de la zone d'étude}

Le bassin versant de la Baya est localisé à l'Est de la Côte d'Ivoire entre les longitudes $2^{\circ} 38^{\prime}$ et $3^{\circ} 33^{\prime} \mathrm{W}$ et les latitudes $6^{\circ} 35^{\prime}$ et $8^{\circ} 26^{\prime} \mathrm{N}$ (Figure1). Il couvre une superficie d'environ $6324 \mathrm{~km}^{2}$. La population du bassin est estimée à 267263 habitants avec un accroissement annuel de $2,8 \%$ de la population (INS, 2014).

Du point de vue pédologique, ce bassin est couvert de sols ferralitiques occupés par des cultures de rente et d'exportation (café, cacao, anacarde) et les cultures vivrières (Mangoua, 2013).Les principales formations géologiques rencontrées peuvent être regroupées en trois grands ensembles lithologiques (Youan Ta et al., 2015). Un ensemble tarkwaien et volcano-sédimentaire constitué principalement de Schiste, d'Amphibolite et de Métadolérite. Au plan hydrogéologique, l'on distingue deux types d'aquifères dans la zone d'étude. Ce sont les aquifères d'altérites et les aquifères de fissures et de fractures. Les premiers sont des réservoirs résultant des processus d'altération physicochimiques et d'érosion du socle. Ils sont composés de sables argileux et d'arènes grenues et constituent le premier niveau de réservoirs en milieu de socle. Ces aquifères sont directement alimentés par les eaux des précipitations. Les seconds se développent dans les zones broyées et/ou fissurées du socle. Ainsi, leur potentialité en eau est liée à la densité de la fracturation du socle (Biémi, 1992). 


\section{Echantillonnage et méthodes analytiques}

L'échantillonnage des eaux souterraines a été effectué en août 2016 et a porté sur 13 forages et 12 puits. Ces points d'eau ont été localisés par leurs coordonnées géographiques déterminées par un GPS. La température $\left(\mathrm{T}{ }^{\circ} \mathrm{C}\right)$, le $\mathrm{pH}$, la conductivité électrique $(\mathrm{CE})$ et l'oxygène dissous $\left(\mathrm{O}_{2}\right)$ ont été mesurés in situ à l'aide d'un multiparamètre de marque WWT 82362 . La turbidité a été également mesurée sur le terrain avec un turbidimètre de marque WTW $315 \mathrm{i}$ (de précision \pm 1 ). Les échantillons ont été conservés à $4{ }^{\circ} \mathrm{C}$ dans une glacière pendant le transport au laboratoire.

Les ions $\mathrm{HCO}_{3}{ }^{-}, \mathrm{Ca}^{2+}, \mathrm{Mg}^{2+}$ et $\mathrm{Cl}^{-}$ont été dosés par la méthode de titration avec l'acide chlorhydrique $0,1 \mathrm{~N}$ à partir d'un Titrateur digital (HACH 1690001). Quant aux autres ions $\mathrm{NO}_{3}^{-}, \mathrm{SO}_{4}{ }^{2-}, \mathrm{Fe}^{2+}, \mathrm{K}^{+}, \mathrm{Na}^{+}, \mathrm{Mn}^{2+}$, $\mathrm{PO}_{4}{ }^{3-}$ et $\mathrm{SiO} 2$, ils ont été dosés selon les méthodes $\mathrm{HACH}$ à l'aide d'un spectrophotomètre UV-Vis DR 6000.

\section{Analyses statistiques}

\section{Evaluation de la qualité des eaux}

Le test de Kruskal-Wallis a été utilisé pour comparer la qualité physicochimique des eaux des puits et forages. Le test de Kruskal-
Wallis $(\mathrm{p}>0,05)$ et la statistique descriptive ont été réalisés par le logiciel Statistica 7,1. L'évaluation de la qualité des eaux est basée sur la comparaison des valeurs des paramètres d'analyses avec celles recommandées par l'OMS (2011) pour l'eau destinée à la consommation.

\section{Acquisition de la minéralisation des eaux}

Les paramètres physicochimiques des eaux de forages et de puits ont été soumis à une analyse en composante principale (ACP) pour déterminer les différents processus intervenant dans la minéralisation des eaux souterraines de la zone d'étude. Cette analyse définit les facteurs principaux dont la corrélation avec les variables permet une explication des phénomènes mis en jeu. L'utilisation optimale de l'ACP nécessitant la normalisation de la distribution des données, celle-ci a été vérifiée par le test statistique HSD (Honest Significant Difference) de Tukey et de corrélation de Pearson. Pour ces tests, trois niveaux de significativité de $\mathrm{p}$ ont été retenus : le niveau significatif $(p<0,05)$, le niveau très significatif $(\mathrm{p}<0,01)$ et le niveau très hautement significatif $(p<0,001)$. Aussi, l'analyse du diagramme de Piper a permis de mettre en exergue les différentes faciès chimique des eaux.

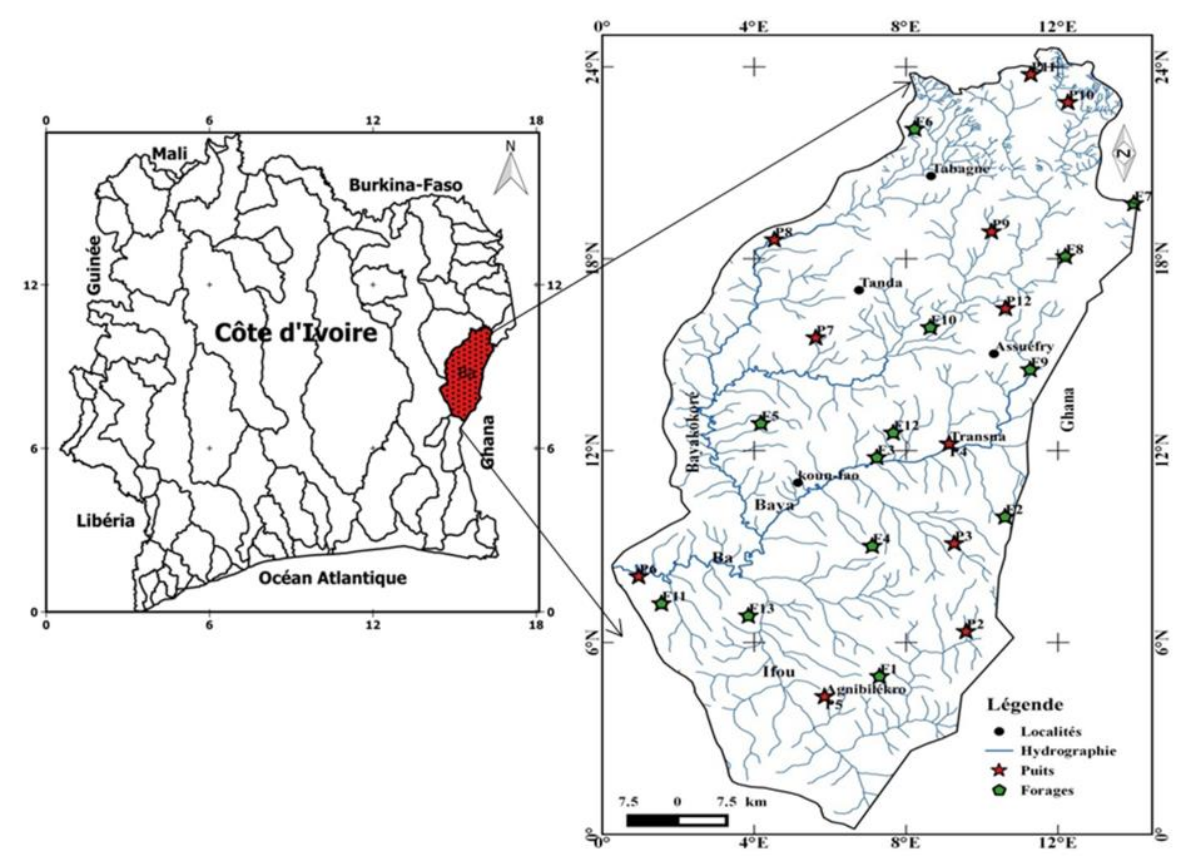

Figure 1: Situation géographique du bassin de la Baya et présentation du réseau des points d'échantillonnage d'eau. 


\section{RESULTATS \\ Qualité physico-chimique des eaux souterraines}

Les résultats des paramètres physicochimiques des eaux échantillonnées sont reportés dans le Tableau 1. Les valeurs moyennes de la température, du $\mathrm{pH}$, de la turbidité de l'oxygène dissous et de la conductivité électrique sont respectivement de $27,07 \pm 0,76 \mathrm{mg} \cdot \mathrm{L}^{-1}, 5,89 \pm 0,86 \mathrm{mg} \cdot \mathrm{L}^{-1}, 8,12$ $\pm 0,8 \mathrm{mg} \cdot \mathrm{L}^{-1}, 3,59 \pm 1,27 \mathrm{mg} \cdot \mathrm{L}^{-1}$ et $455 \pm$ $348,47 \mu{\mathrm{S} . \mathrm{cm}^{-1}}^{-1}$ pour les eaux de forage et de $27,14 \pm 0,97 \mathrm{mg} . \mathrm{L}^{-1}, 6,08 \pm 0,84 \mathrm{mg} . \mathrm{L}^{-1}, 36,38$ $\pm 8,27 \mathrm{mg} . \mathrm{L}^{-1}, 4,57 \pm 1,69 \mathrm{mg} . \mathrm{L}^{-1}$ et $347,96 \pm$ $304,91 \mu \mathrm{S} . \mathrm{cm}^{-1}$ pour les eaux de puits. Toutefois aucune différence significative n'est observée pour ces paramètres à l'exception de la turbidité qui est significativement plus élevée $(\mathrm{p}>0,05)$ dans les eaux de puits.

Les valeurs moyennes des teneurs en magnésium, potassium, sodium, en silice et en sulfate ne présentent aucune différence significative $(p>0,05)$ dans les eaux de forage et celle des puits.

L'ammonium et le calcium par contre présentent une différence significative ( $p>$ $0,05)$ entre les forages et puits avec des teneurs moyennes respectives de 0,23 $\pm 0,07$ et $0,44 \pm 0,18 \mathrm{mg} . \mathrm{L}^{-1}$ pour l'ammonium et $115,9 \pm 38,31$ et $68,58 \pm 32,25 \mathrm{mg} . \mathrm{L}^{-1}$ pour le calcium. Les eaux de puits sont donc plus chargées en ammonium, contrairement à celles des forages plus riches en calcium.

$\mathrm{Au}$ plan spatial (Figure 2), les valeurs élevées pour la conductivité électrique sont observées dans le puits (P9) (Figure 2a) et dans les forages (F11, F12 et F13) avec des teneurs maximales supérieures à $1000 \mu \mathrm{S} . \mathrm{cm}^{-1}$ (Figure 2c). Les teneurs maximales en chlorures $\left(\mathrm{Cl}^{-}\right)$bien qu'en dessous de la directive OMS, présentent des concentrations élevées dans les forages F11 d'Akrassikro (141,83 mg.L $\left.{ }^{-1}\right)$, F13 de Cpt Kouakou (177,3 mg. $\mathrm{L}^{-1}$ ), de même que dans les puits P3 de kouassia-nanguini (114 mg. $\mathrm{L}^{-1}$ ) et P9 de Tiédo (123 mg. $\mathrm{L}^{-1}$ ) (Figure $2 \mathrm{~b}$ et $2 \mathrm{~d}$ ). Les orthophosphates $\left(\mathrm{PO}_{4}{ }^{3-}\right)$ enregistrent des concentrations faibles aussi bien dans les puits que dans les forages à l'exception du puits P5 d'Agnibilékro (5,23 mg. $\left.\mathrm{L}^{-1}\right)$ qui enregistre une concentration supérieure à la directive OMS qui est de 5 mg.L ${ }^{-1}$ (Figure $2 \mathrm{~d}$ ).

Les concentrations en nitrates $\left(\mathrm{NO}_{3}{ }^{-}\right)$ enregistrées dans le forage (F10) de Siédja
(109,6 mg.L $\left.\mathrm{L}^{-1}\right)$ et dans les puits de Kouassiananguini (P3), Agnibilekro (P5) et Tiédo (P9) respectivement $\left(79,5 ; 110,8 ; 56,7 \quad \mathrm{mg} . \mathrm{L}^{-1}\right)$ dépassent largement la valeur recommandée par la directive OMS qui est de $50 \mathrm{mg} . \mathrm{L}^{-1}$ (Tableau 2).

Les éléments métalliques en trace que sont le Fer $\left(\mathrm{Fe}^{2+}\right)$ et le Manganèse $\left(\mathrm{Mn}^{2+}\right)$ ont des concentrations hétérogènes. Environ $38 \%$ des localités visitées affichent des teneurs nulles en fer et en manganèse. Cependant, $23 \%$ des échantillons dosés présentent à la fois des teneurs élevées en fer et en manganèse supérieures à la directive OMS (Tableau 3).

\section{Processus de minéralisation \\ Faciès Hydrogéochimique}

Le faciès hydrogénocarbonaté calcique et magnésien domine les eaux souterraines du bassin (soit 68\%). Le faciès chloruré calcique et magnésien ne présente que $32 \%$ des échantillons d'eau du bassin (Figure 3). L'ordre d'importance des principaux ions dans les eaux souterraines de la région se résume comme suit : $\mathrm{Ca}^{2+}>\mathrm{Mg}^{2+}>\mathrm{Na}^{+}>\mathrm{K}^{+}$ pour les cations et $\mathrm{HCO}_{3}{ }^{-}>\mathrm{Cl}^{-}>\mathrm{SO}_{4}{ }^{2-}>\mathrm{NO}_{3}$ pour les anions.

\section{Analyse en composantes principales (ACP)}

L'analyse des résultats de l'ACP a permis également de calculer les valeurs propres et les variances exprimées pour chaque facteur et leur cumul (Tableau 4). Le pourcentage de variances exprimées est de $21,28 \%$ pour le facteur $1 ; 18,24 \%$ pour le facteur $\mathrm{F} 2 ; 14,54 \%$ pour le facteur 3 et de $10,22 \%$ pour le facteur 4 . La représentation à l'aide de ces quatre facteurs rend compte de manière satisfaisante, la structure des nuages de points. Le cumul de la variance exprimée pour les quatre facteurs est de $64,28 \%$.

\section{Corrélation entre les variables}

Le Tableau 5 montre que la conductivité électrique (CE) est significativement $(\mathrm{P}<0,01)$ corrélée avec l' $\mathrm{O}_{2}$ $(0,48)$ et $\mathrm{Cl}^{-1}(0,68)$. La turbidité est fortement corrélée $(\mathrm{P}<0,001)$ à l'ammonium $(0,62)$ et à un degré moindre au manganèse $(0,45)$. Le $\mathrm{pH}$ quant à lui est corrélé faiblement $(\mathrm{P}<0,05)$ à l'hydrogénocarbonate et négativement à l'ammonium et au manganèse. Les ions $\mathrm{HCO}_{3}{ }^{-}$présentent une forte $(\mathrm{P}<0,001)$ 
corrélation avec $\mathrm{Mg}^{2+}(0,62)$ qui lui est significativement corrélé à $\mathrm{Ca}^{2+}(0,68)$. Cependant, ces deux derniers sont corrélés moyennement avec $\mathrm{Na}^{+}$. Quant à $\mathrm{K}^{+}$, il est fortement corrélé à $\mathrm{Mn}^{2+}(0,59)$. Les ions $\mathrm{Fe}^{2+}$ et $\mathrm{Mn}^{2+}$ ne présentent aucune corrélation significative. Par ailleurs, le manganèse est significativement $(\mathrm{P}<0,001)$ corrélé avec $\mathrm{NH}_{4}^{+}(0,62)$, moyennement avec $\mathrm{PO}_{4}{ }^{3-}$ et négativement avec $\mathrm{O}_{2}(-0,46)$. Il n'existe aucune corrélation entre les composés azotés $\left(\mathrm{NO}_{3}{ }^{-}\right.$et $\left.\mathrm{NH}_{4}{ }^{+}\right)$, cependant, ces deux éléments sont moyennement corrélés $(\mathrm{P}<0,01)$ au orthophosphate avec un coefficient de corrélation autour de 0,5 .

\section{Analyse des variables dans les plans factoriels}

L'analyse du cercle de communauté du plan factoriel F1-F2 (Figure 4a) montre que le facteur F1, est commandé dans sa partie positive par les variables $\mathrm{NH}_{4}{ }^{+}, \mathrm{NO}_{2}{ }^{-}, \mathrm{PO}_{4}{ }^{3-}$, $\mathrm{SO}_{4}{ }^{2-}, \mathrm{Cl}^{-}$,Turb, et $\mathrm{O}_{2}$; quant au facteur $\mathrm{F} 2$, il est commandé dans sa partie positive par les variables $\mathrm{HCO}_{3}^{-}, \mathrm{Ca}^{2+}, \mathrm{Mg}^{2+}, \mathrm{Na}^{+}, \mathrm{SiO} 2$ et $\mathrm{Mn}^{2+}$.Ces derniers, sont constitués en majorité d'eaux de forages, tandis que les localités de Transua, Tiédo, Kouassia-nanguini et Gouméré) (Figure 4b) présentées sur la partie positive de l'axe F1, sont constituées uniquement d'eaux de puits.

Tableau 1: Statistique descriptive et de comparaison entre les paramètres physico-chimiques des eaux des puits et forages du bassin versant de la Baya.

\begin{tabular}{|c|c|c|c|c|c|c|c|}
\hline \multirow[t]{2}{*}{ Paramètres } & \multicolumn{3}{|c|}{ Forages $(n=13)$} & \multicolumn{3}{|c|}{ Puits $(n=12)$} & \multirow{2}{*}{$\begin{array}{l}\text { OMS } \\
\text { (2011) }\end{array}$} \\
\hline & $\operatorname{Moy} \pm \sigma$ & $\max$ & $\min$ & $\operatorname{Moy} \pm \sigma$ & $\max$ & $\min$ & \\
\hline $\mathbf{T}^{\circ} \mathbf{C}$ & $27,07^{\mathrm{a}} \pm 0,76$ & 28,3 & 25,9 & $27,14^{\mathrm{a}} \pm 0,97$ & 28,3 & 25,1 & $25-30$ \\
\hline pH & $5,89^{\mathrm{a}} \pm 0,86$ & 6,98 & 4,06 & $6,08^{\mathbf{a}} \pm 0,84$ & 7,73 & 5,17 & $6,5-8,5$ \\
\hline Turb (UNT) & $8,12^{\mathrm{a}} \pm 0,8$ & 33,8 & 1,61 & $36,38^{\mathbf{b}} \pm 8,27$ & 130 & 4,43 & $<15$ \\
\hline $\mathbf{C E}\left(\mu \mathrm{S} . \mathrm{cm}^{-1}\right)$ & $455^{\mathrm{a}} \pm 348,47$ & 1236 & 28,9 & $347,96^{\mathbf{a}} \pm 304,91$ & 1092 & 83,6 & - \\
\hline $\mathbf{H C O}_{\mathbf{3}}^{-}\left(\mathrm{mg} \cdot \mathrm{L}^{-1}\right)$ & $196,36^{\mathbf{a}} \pm 134,01$ & 489,6 & 60,8 & $143,03^{a} \pm 137,22$ & 489,6 & 25,2 & - \\
\hline $\mathbf{C a}^{2+}\left(\mathrm{mg} \cdot \mathrm{L}^{-1}\right)$ & $115,9^{\mathrm{a}} \pm 38,31$ & 162 & 42,82 & $68,58^{\mathbf{b}} \pm 32,25$ & 146 & 28 & - \\
\hline $\mathbf{M g}^{2+}\left(\mathrm{mg} \cdot \mathrm{L}^{-1}\right)$ & $66,3^{a} \pm 42,6$ & 145 & 23 & $48,42^{\mathrm{a}} \pm 36,34$ & 145 & 14 & - \\
\hline $\mathbf{N a}^{+}\left(m g \cdot L^{-1}\right)$ & $5,56^{\mathrm{a}} \pm 4,6$ & 12,1 & 0,99 & $4,79^{a} \pm 2,58$ & 9,9 & 1,6 & - \\
\hline $\mathbf{k}^{+}\left(\mathrm{mg} \cdot \mathrm{L}^{-1}\right)$ & $1,88^{\mathbf{a}} \pm 1,89$ & 5,8 & 0,4 & $3,74^{\mathrm{a}} \pm 8,02$ & 28,8 & 0,5 & - \\
\hline $\mathbf{C l}^{-}\left(\mathrm{mg} \cdot \mathrm{L}^{-1}\right)$ & $58,09^{a} \pm 101,43$ & 338,1 & 2 & $30,67^{\mathrm{a}} \pm 41,84$ & 123 & 3 & $\leq 250$ \\
\hline $\mathbf{F e}^{2+}\left(\mathrm{mg} . \mathrm{L}^{-1}\right)$ & $2,73^{\mathrm{a}} \pm 5,07$ & 16 & 0,09 & $1,05^{\mathbf{a}} \pm 1,46$ & 4,56 & 0,02 & $<0,3$ \\
\hline $\mathbf{M n}^{2+}\left(\mathrm{mg} \cdot \mathrm{L}^{-1}\right)$ & $0,07^{\mathrm{a}} \pm 0,08$ & 0,22 & 0,02 & $0,19^{\mathbf{a}} \pm 0,21$ & 0,56 & 0,03 & $<0,05$ \\
\hline $\mathbf{O}_{2}\left(\mathrm{mg} \cdot \mathrm{L}^{-1}\right)$ & $3,59^{\mathrm{a}} \pm 1,27$ & 5,17 & 1,56 & $4,57^{\mathrm{a}} \pm 1,69$ & 7,68 & 2,5 & \\
\hline $\mathbf{N O}_{\mathbf{3}}^{-}\left(\mathrm{mg} \cdot \mathrm{L}^{-1}\right)$ & $21,43^{\mathrm{a}} \pm 31,78$ & 109,6 & 0 & $23,59^{a} \pm 37,55$ & 110,8 & 0,6 & $\geq 50$ \\
\hline $\mathbf{N H}_{\mathbf{4}}{ }^{+}\left(\mathrm{mg} \cdot \mathrm{L}^{-1}\right)$ & $0,23^{\mathbf{a}} \pm 0,07$ & 0,42 & 0,16 & $0,44^{\mathbf{b}} \pm 0,18$ & 0,81 & 0,23 & 1,5 \\
\hline $\mathbf{P O}_{4}{ }^{3-}\left(\mathrm{mg} \cdot \mathrm{L}^{-1}\right)$ & $1,05^{\mathrm{a}} \pm 0,65$ & 2,7 & 0,37 & $1,72^{\mathrm{a}} \pm 1,57$ & 5,23 & 0,51 & $<5$ \\
\hline $\mathrm{SiO2}\left(\mathrm{mg} . \mathrm{L}^{-1}\right)$ & $33,9^{\mathrm{a}} \pm 21,2$ & 68 & 2 & $41,42^{\mathrm{a}} \pm 27,33$ & 99 & 12 & $\leq 10$ \\
\hline $\mathbf{S O}_{4}{ }^{2-}\left(\mathrm{mg} \cdot \mathrm{L}^{-1}\right)$ & $1,1^{\mathrm{a}} \pm 0,73$ & 2 & 0 & $6,5^{a} \pm 9,48$ & 32 & 1 & $\leq 250$ \\
\hline
\end{tabular}

Test de Kruskal-wallis à p > 0,05. Pour un paramètre donné, il n’y a pas de différence significative portant une même lettre a ou b. 
Tableau 2 : Localités ayant des teneurs $\mathrm{NO}_{3}{ }^{-}$et $\mathrm{PO}_{4}{ }^{3-}$ supérieures à la directive OMS (2011).

\begin{tabular}{lcccc}
\hline Localités & Siédja (F10) & Agnibilekro (P5) & K-nangrin (P3) & Tiédo (P9) \\
\hline X coordonnée & 492395 & 474140 & 496050 & 468667 \\
Y coordonnée & 855807 & 783904 & 816652 & 871727 \\
\hline $\mathrm{NO}_{3}{ }^{-}\left(\mathrm{mg} . \mathrm{L}^{-1}\right)$ & 109,6 & 110,8 & 79,5 & 56,7 \\
$\mathrm{PO}_{4}{ }^{3-}\left(\mathrm{mg} . \mathrm{L}^{-1}\right)$ & 2,7 & 5,23 & 4,4 & 2,47 \\
\hline
\end{tabular}

Tableau 3 : Localités ayant des teneurs en $\mathrm{Fe}^{2+}$ et $\mathrm{Mn}^{2+}$ supérieures à la directive OMS (2011).

\begin{tabular}{|c|c|c|c|c|c|c|}
\hline Localités & Dua-kouamé & Kinkua & K-nangrin & Transua & kékéreni & Guimini \\
\hline $\mathrm{Fe}^{2+}\left(\mathrm{mg} . \mathrm{L}^{-1}\right)$ & 6,84 & 16 & 4,56 & 1,65 & 15 & 1,373 \\
\hline $\mathrm{Mn}^{2+}\left(\mathrm{mg} . \mathrm{L}^{-1}\right)$ & 0,218 & 0,019 & 0,563 & 0,563 & 1 & 2,42 \\
\hline
\end{tabular}

Tableau 4: Valeurs propres et pourcentages exprimés par les axes principaux.

\begin{tabular}{lcccc}
\hline Variables & F1 & F2 & F3 & F4 \\
\hline Valeur Propre & 4,25 & 3,65 & 2,91 & 2,04 \\
\% de Variance exprimée & 21,28 & 18,24 & 14,54 & 10,22 \\
Cumul de valeur propre & 4,25 & 7,90 & 10,81 & 12,85 \\
\% de Variance exprimée cumulée & 21,28 & 39,52 & 54,06 & 64,28 \\
\hline
\end{tabular}

Tableau 5: Matrice de corrélation des paramètres physico-chimiques des eaux souterraines du bassin versant de la Baya.

\begin{tabular}{|c|c|c|c|c|c|c|c|c|c|c|c|c|c|c|c|}
\hline & pH & Turb & $\mathbf{C E}$ & $\mathrm{HCO}_{3}^{-}$ & $\mathrm{Ca}^{2+}$ & $\mathrm{Mg}^{2+}$ & $\mathrm{Na}^{+}$ & $\mathbf{k}^{+}$ & $\mathrm{Cl}^{-}$ & $\mathrm{Fe}^{2+}$ & $\mathrm{Mn}^{2+}$ & $\mathrm{O}_{2}$ & $\mathrm{NO}_{3}{ }^{-}$ & $\mathrm{NH}_{4}^{+}$ & $\mathrm{PO}_{4}{ }^{3-}$ \\
\hline pH & 1 & & & & & & & & & & & & & & \\
\hline Turb & 0,11 & 1 & & & & & & & & & & & & & \\
\hline $\mathbf{C E}$ & $0,53 * *$ & 0,06 & 1 & & & & & & & & & & & & \\
\hline $\mathrm{HCO}_{3}^{-}$ & $0,33 *$ & $-0,08$ & 0,27 & 1 & & & & & & & & & & & \\
\hline $\mathrm{Ca}^{2+}$ & $-0,14$ & 0,02 & 0,15 & $0,54 * *$ & 1 & & & & & & & & & & \\
\hline $\mathbf{M g}^{2+}$ & $-0,03$ & 0,18 & $-0,08$ & $0,62 * * *$ & $0,68 * * *$ & 1 & & & & & & & & & \\
\hline $\mathrm{Na}^{+}$ & $-0,12$ & 0,13 & $-0,01$ & $0,41 *$ & $0,49 * *$ & $\mathbf{0 , 5 2} * *$ & 1 & & & & & & & & \\
\hline $\mathbf{k}^{+}$ & $-0,20$ & $0,36^{*}$ & 0,03 & 0,10 & 0,08 & 0,13 & 0,22 & 1 & & & & & & & \\
\hline $\mathrm{Cl}^{-}$ & 0,15 & $-0,01$ & $0,68 * *$ & 0,02 & 0,18 & $-0,11$ & $-0,09$ & $-0,07$ & 1 & & & & & & \\
\hline $\mathrm{Fe}^{2+}$ & 0,06 & 0,08 & $-0,08$ & $-0,01$ & 0,16 & 0,23 & $-0,13$ & 0,01 & $-0,05$ & 1 & & & & & \\
\hline $\mathrm{Mn}^{2+}$ & $-0,37 *$ & $0,45 * *$ & $-0,16$ & 0,14 & 0,23 & $\mathbf{0 , 4 3} *$ & 0,28 & $0,59 * * *$ & $-0,02$ & 0,18 & 1 & & & & \\
\hline $\mathrm{O}_{2}$ & $0,54 * *$ & $-0,08$ & $0,48 * * *$ & $-0,04$ & $-0,37 *$ & $-0,54 * *$ & $-0,26$ & $-0,13$ & $0,32 *$ & $-0,24$ & $-0,46^{* *}$ & 1 & & & \\
\hline $\mathrm{NO}_{3}^{-}$ & $-0,16$ & $-0,04$ & $-0,09$ & $-0,06$ & 0,24 & 0,22 & $-0,04$ & $-0,06$ & 0,04 & $-0,04$ & 0,009 & $-0,18$ & 1 & & \\
\hline $\mathbf{N H}_{4}^{+}$ & $-0,31 *$ & $0,62 * * *$ & $-0,25$ & $-0,05$ & 0,19 & $0,48 * *$ & 0,29 & 0,29 & $-0,04$ & 0,20 & $0,62 * * *$ & $-0,62 * * *$ & 0,03 & 1 & \\
\hline $\mathrm{PO}_{4}{ }^{3-}$ & $-0,21$ & $0,36^{*}$ & $-0,16$ & $-0,19$ & 0,18 & $0,32 *$ & 0,13 & 0,00 & 0,04 & 0,12 & $0,41 * *$ & $-0,37 *$ & $0,47 * *$ & $0,52 * *$ & 1 \\
\hline
\end{tabular}



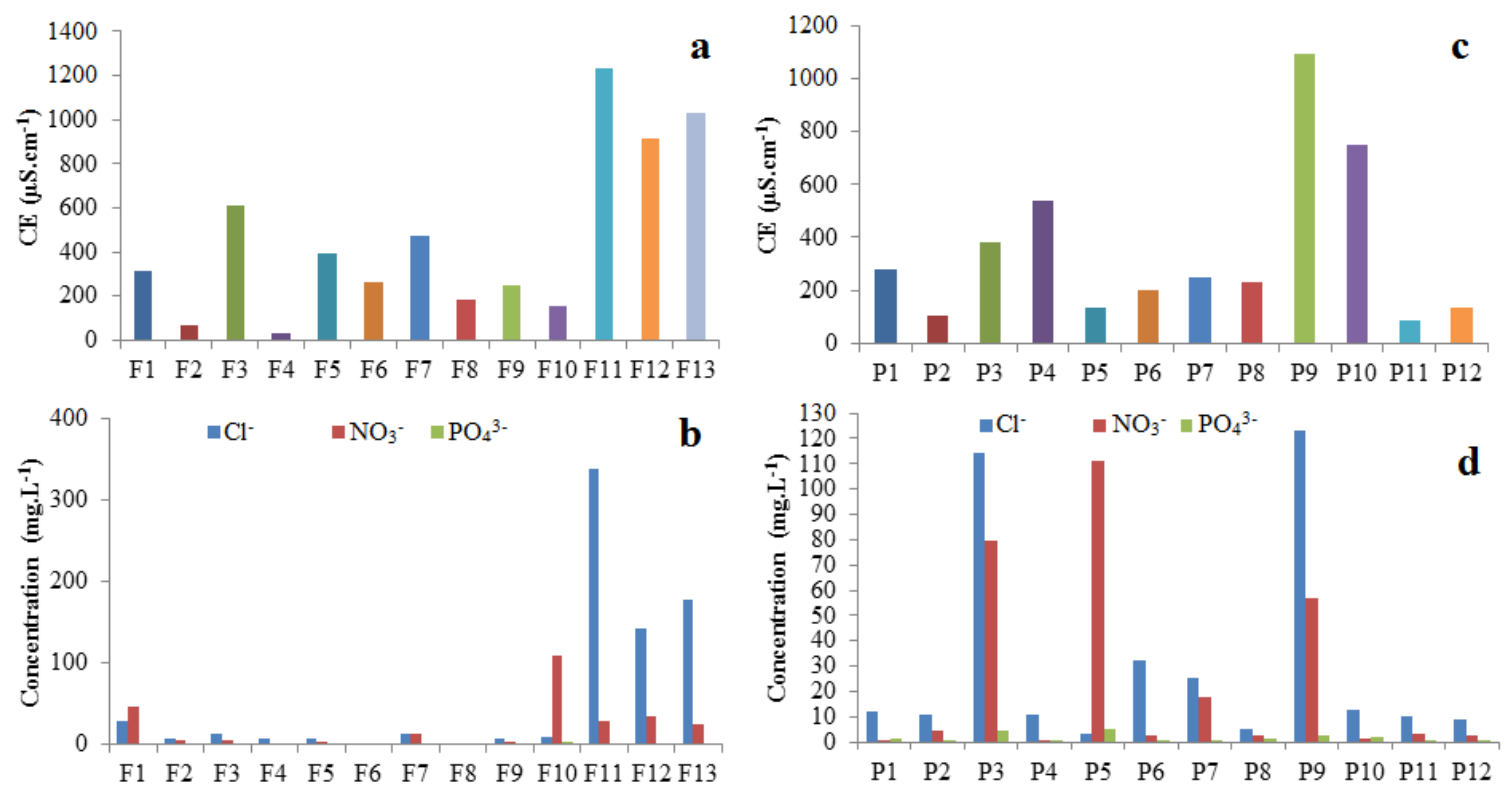

Figure 2: Variation de la conductivité électrique, du nitrate, du phosphate et des chlorures dans les puits $(\mathrm{P})$ et forages $(\mathrm{F})$ du bassin versant de la Baya.

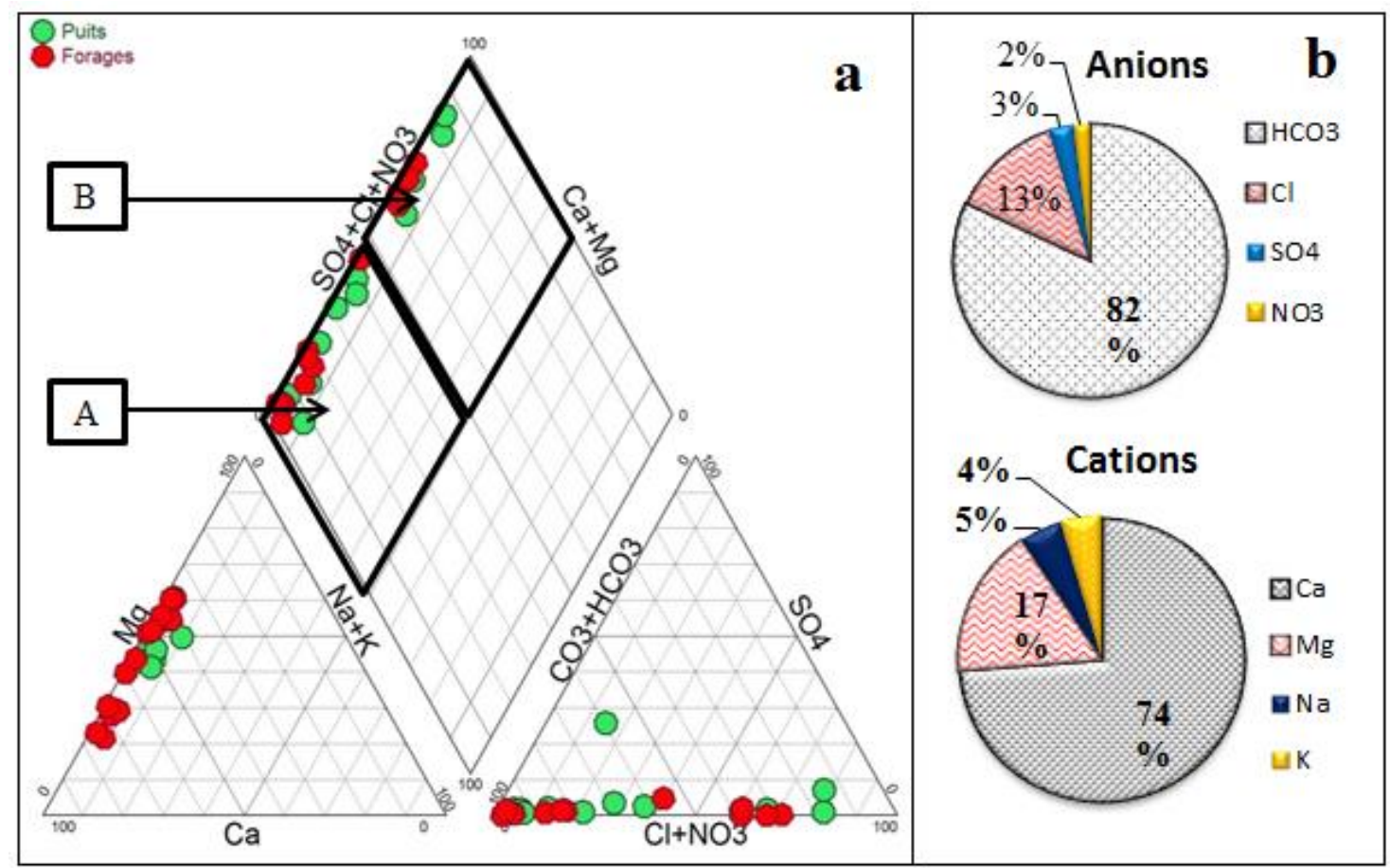

A: Faciès hydrogénocarbonatés calciques et magnésiens B: Faciès chlorurés calciques et magnésiens.

Figure 3: Présentation des eaux dans le diagramme de Piper (a) et circulaire (b). 
G. K. N'GUETTIA et al. / Int. J. Biol. Chem. Sci. 13(1): 574-585, 2019
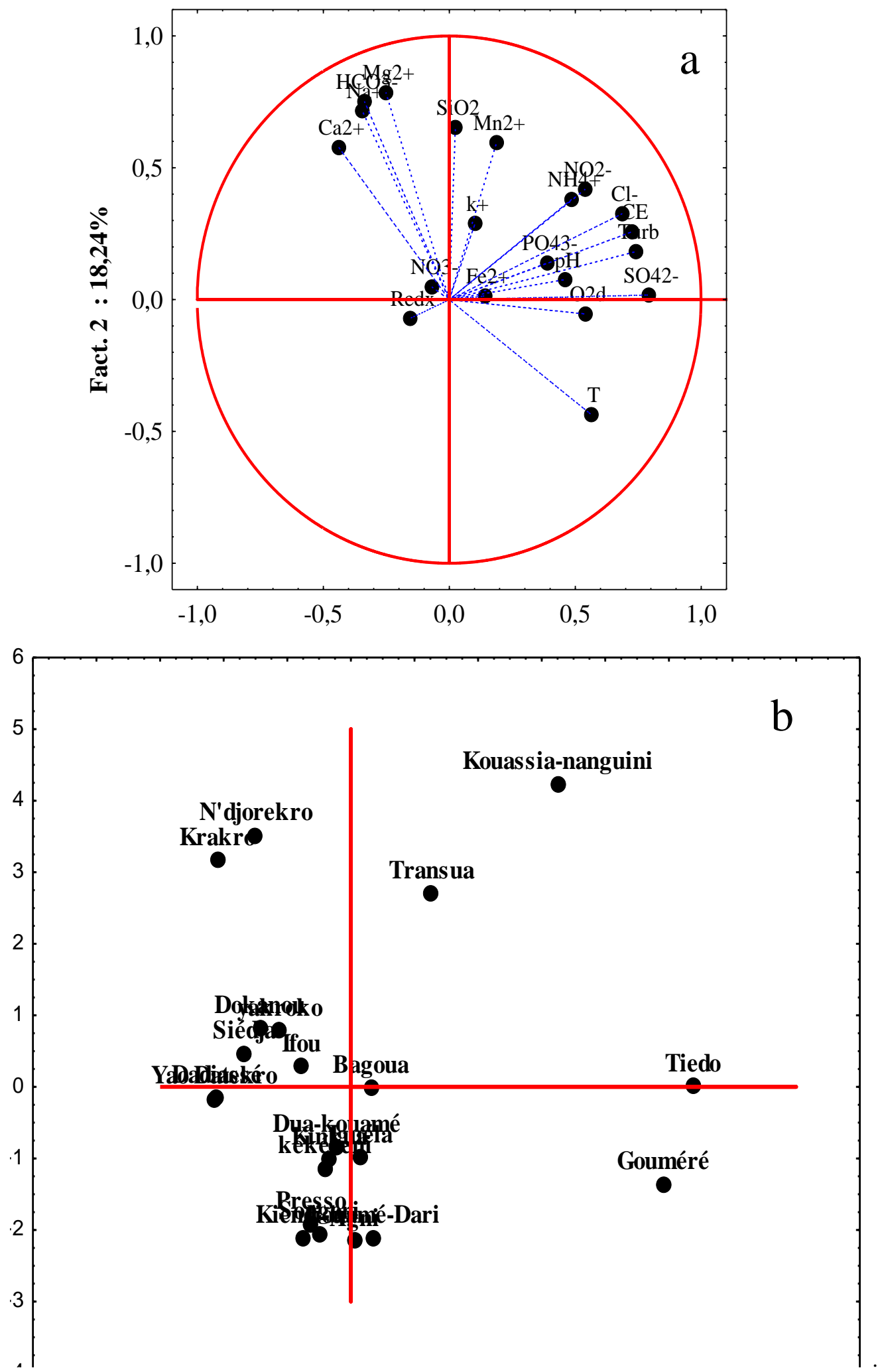

Figure 4: Analyse dans l'espace des variables et des individus (plan factoriel F1 - F2) 


\section{DISCUSSION}

La comparaison des concentrations des paramètres physico-chimiques à la directive de l'OMS (2011) pour une eau potable, permet de faire certaines remarques. Les valeurs moyennes (conductivité, $\mathrm{pH}$, sodium, magnésium, potassium, température et chlorures) sont dans les limites préconisées par la directive. Cependant, plusieurs eaux de puits et forages en certains points présentent des concentrations largement au-dessus de la limite préconisée par l'OMS (2011). Ce sont les nitrates $(9,52 \%)$, l'orthophosphate $(50 \%)$, le manganèse et le fer $(23,96 \%)$, la silice $(97,61 \%)$ et la turbidité $(1,78 \%)$.Ces résultats indiquent la détérioration de la qualité physico-chimique des eaux. En effet, les travaux de Abbou et al. (2014) au Maroc et Heriarivony et al. (2016) à Madagascar ont respectivement montrés que les teneurs élevées de nitrates et orthophosphates des eaux de puits et forages témoignent d'une pollution chimique récente et d'origine humaine. Par ailleurs, la consommation de ces eaux pourrait affecter la santé humaine. Ainsi les concentrations anormales de nitrates rencontrées dans certains forages et puits peuvent occasionner la méthémoglobinémie chez le nourrisson et des maladies cancérigènes chez l'adulte (Cidu et al., 2009). Les teneurs élevées en silice dans l'eau de consommation, comme c'est le cas dans la zone d'étude pourraient avoir pour conséquences des manifestations pathologiques au niveau du poumon (Eblin et al., 2014). Les fortes teneurs en fer et en manganèse entrainent un problème d'acceptabilité de l'eau par les populations. En effet, ces deux éléments confèrent à l'eau un goût métallique désagréable. Selon Goné et al. (2005), les fortes teneurs de $\mathrm{Fe}^{2+}$ et $\mathrm{Mn}^{2+}$ suggèrent que le milieu est pauvre en $\mathrm{O}_{2}$ dissous.

Dans l'ensemble, les eaux sont acides et faiblement minéralisées, avec une prédominance en ion hydrogénocarbonate
$\left(\mathrm{HCO}_{3}{ }^{-}\right)$et calcium $\left(\mathrm{Ca}^{2+}\right)$ respectivement à $82 \%$ et $74 \%$. Cette prédominance est une caractéristique majeure des eaux de socle en milieu cristallin et cristallophyllien. Des travaux effectués dans d'autres régions de la Côte d'Ivoire en milieu de socle notamment dans la région d'Odienné (Kouassi et al., 2017) et dans le bassin transfrontalier de la Comoé (Ouattara et al., 2016) mettent également en évidence l'importance du faciès hydrogénocarbonate. Par ailleurs, cette minéralisation des eaux est plus prononcée dans les forages que dans les puits. Cette différenciation pourrait traduire un temps de résidence plus prolongé pour les eaux de forages, plus profondes par rapport aux eaux de puits. (Ligban et al., 2009). En effet, selon Ahoussi et al. (2012), la présence des ions calcium et hydrogénocarbonates dans l'eau proviendrait de l'altération des carbonates $\left(\mathrm{CaCO}_{3}\right)$ d'après la formule suivante:

$\mathrm{CaCO}_{3(\mathrm{~S})}+\mathrm{H}_{2} \mathrm{O}_{(\mathrm{l})}+\mathrm{CO} 2(\mathrm{~g})$

$\mathrm{Ca}^{2+}+2 \mathrm{HCO}_{3}^{-}$(aq) (1).

En effet, c'est cette réaction qui explique la dominance $\mathrm{du}$ faciès hydrogénocarbonaté calcique dans les eaux du bassin. A la différence des eaux de forages, plus représentatives dans la partie positive du plan factoriel $F_{2}$, la partie positive du plan $F_{1}$ est marquée par les eaux de puits qui sont chlorurées et nitratées. Ce qui dénote l'existence d'une différenciation chimique entre les nappes échantillonnées. L'importance des ions $\mathrm{Cl}^{-}, \mathrm{NO}_{3}{ }^{-}, \mathrm{PO}_{4}{ }^{2-}$ et $\mathrm{NH}_{4}{ }^{+}$dans les puits est à relier à une origine anthropique et biogénique. Ces ions proviendraient des effluents domestiques et agricoles, liées aux activités humaines (Heriarivony et al., 2016). La position superficielle des réservoirs captés par les puits se traduit également par des valeurs élevées de turbidité qui est une mesure globale qui prend en compte toutes les matières, soit colloïdales, soit insolubles, d'origine minérale ou organique (Béchir et al., 2007). Ces résultats corroborent avec ceux de Shorieh et al. (2015) 
et de Ahoussi et al (2012) obtenus dans les eaux souterraines de Poitiers et dans la région de Bondoukou où la source principale de nitrates dans les eaux de puits est attribuable à des activités humaines. Le phénomène est plus sensible dans les puits des localités de Tiedo, de Kouassia-naguini et d'Agnibilekro où les concentrations en nitrates sont supérieures à la directive OMS (2011) qui est de $50 \mathrm{mg} . \mathrm{L}^{-1}$. Ce résultat vient étayer les études antérieures de Mangoua (2013) qui ont montré que les eaux souterraines du bassin sont influencées par la pollution à cause de la forte pression anthropique. Par ailleurs, les travaux de Aké et al. (2012) et Blé et al. (2016) ont montrés que les eaux souterraines ont atteint un niveau de pollution inquiétant en Côte d'Ivoire et en Afrique en particulier. Ainsi, d'importantes teneurs en nitrates, supérieures à la directive OMS ont-elles été mises en évidence dans les eaux souterraines au Nigéria (Adelam, 2006), au Ghana (Kortatsi et al., 2007), au Zimbabwe (Nyamangara, 2013) et à Madagascar (Heriarivony et al., 2016).

\section{Conclusion}

La présente étude a été entreprise dans l'objectif d'évaluer la qualité physicochimique et de déterminer les processus à l'origine de la minéralisation des eaux souterraines du bassin versant de la Baya. L'analyse faite aux eaux de puits et forages indique que près de $85 \%$ des eaux échantillonnées sont de bonnes qualités. Cependant, celles échantillonnées dans les localités de Siédjà, Tiedo, Kouassia-naguini et Agnibilekro présentent des concentrations en $\mathrm{NO}_{3}{ }^{-}, \mathrm{Cl}^{-}, \mathrm{NH}_{4}{ }^{+}$et $\mathrm{PO}_{4}{ }^{3-}$ au-dessus de la recommandation OMS. La minéralisation de ces eaux est à la fois le fait de processus naturels (interaction eau-roche et mise en solution de produits d'altération, dissolution) et une minéralisation induite par apports superficiels des eaux domestiques et lessivage des sols agricoles. Une surveillance régulière devrait être effectuée afin d'éviter la consommation d'eau contaminée et ses effets associés sur la santé. L'implantation des fosses septiques à une distance réglementaire des sources d'eau potable.

\section{INTERETS CONCURRENTS}

Les auteurs déclarent ne pas avoir d'intérêts concurrents.

\section{CONTRIBUTIONS DES AUTEURS}

GKN est l'investigateur principal de ce manuscrit; JMOM a contribué à la collecte, à l'analyse des données, à l'interprétation des résultats et rédaction de l'article; NKA et AGD ont participé à l'interprétation des résultats, la lecture et la correction du manuscrit ; GLC a assuré la supervision générale des travaux effectués.

\section{REMERCIEMENTS}

Nous remercions le collège des chercheures du Laboratoire Géosciences et Environnement de l'Unité de Formation et de Recherche en Sciences et Gestion de l'Environnement pour leur apport scientifique.

\section{REFERENCES}

Abbou BM, Fadil F, Elhaji M, Zemzami M. 2014. Impact anthropique sur la qualité des eaux souterraines du bassin versant de l'oued Taza (maroc) Eur. Sc. J.,10 (5):117-137.

DOI: http://dx.doi.org/10.19044/esj.

Adelam SMA. 2006. Nitrates pollution of groundwater in Nigeria. Groundwater pollution in Africa, Editors Yongxin Xu and Brent Usher, Taylor \& Francis/Balkema, Great-Britain, 6(2): $37-45$.

DOI: https://www.researchgate.net/publication /265728828.

Ahoussi KE, Loko S, Adja MG, Lasm T, Jourda JP. 2012. Étude hydrogéochimique des eaux des aquifères de fractures du socle Paléoprotérozoïque du Nord-Est de la 
Côte d'Ivoire : Cas de la région de Bondoukou. Afr. Sci., 08: 51-68. DOI : http://dx.doi.org/10.4314/ijbcs.v10i1.33.

Ake GE, Kouadio BH, AdjaMG, Ettien JB, Effebi KR, Biemi J. 2012. Cartographie de la vulnérabilité multifactorielle à l'érosion hydrique des sols de la région de Bonoua (Sud-Est de la Côte d'Ivoire). Phys. Géol. Rev. Org., 31(1): 157-171. DOI : 10.4000/physio-geo.2285.

Béchir BT, Khalifa R, Houda B. 2007. Elimination de la turbidité par oxygénation et filtration successives des eaux de la station de Sfax (Sud de la Tunisie). Rev. Sci. Eau, 20(4): 355-365. DOI :

https://doi.org/10.5897/IJWREE2013.04 33.

Biemi J. 1992. Contribution à l'étude géologique, hydrogéologique et par télédétection des bassins versants subsaheliens du socle précambrien d'Afrique de l'Ouest: Hydro structurale, hydrodynamique, hydrochimie et isotopie des aquifères discontinus des sillons et aires granitiques de la Haute Marahoué (Côte d'Ivoire). Thèse de Doctorat d'Etat ès Sciences Naturelles, Université Nationale d'Abidjan, Côte d'Ivoire, p. 493.

Ble O, Ake GE, Soro T, Soro G, Kouadio EY. 2016. Evaluation de la Qualité Hydrochimique des Eaux Souterraines de la Région de Bonoua. (Sud-Est de la Côte d'Ivoire). Int. J. Innov Applied Studies, 14: 896-907. DOI : http://www.ijias.issr-journals.org.

Cidu R, Biddau R, Fanfani L. 2009. Impact of past ménin activity on the quality of groundwater in SW Sardinia (Italy). $J$. Geoch. Expl., 100: 125-132. DOI: 10.1007/s12145-009-0019-6.

Eblin SG, Soro GM, Sombo AP, Aka N, Kambiré O. 2014. Hydrochimie des eaux souterraines de la région d'Adiaké (sud- est Côte d'Ivoire). Larhy J., 17: 193-214. DOI : 10.4236/Nr.2015.611050.

Goné DL, Savané I, N'go YA, Biémi J. 2005. Mobilité relative des cations majeurs lors de l'altération des roches et acquisition de la minéralisation des eaux souterraines dans le degré carré de Man. Sci. Nat., 2(1): 85-94. DOI : https://doi.org/10.5897/AJAR2015.1021 2.

Heriarivony SC, Razanamparany B, Rakotomalala JE. 2016. Variations spatio-temporelles des paramètres physico-chimiques des eaux souterraines de la commune rurale d'Antanifotsy, Vakinankaratra, Madagascar. Larhy. J., 27:

239-255.

DOI :http://creativecommons.org/license s/by/4.0.

INS. 2014. Institut National de la Statistique, Recensement général de la Population et de l'Habitat. www. ins.ci.,p. 153.

Kouassi WF, Mangoua JM, Kouassi KA, Kouadio ZA, Alle PH, Goula BTA. $2017 . \quad$ Caractéristiques hydrogéochimiques des aquifères fissurés de la région d'Odienné, NordOuest de la Côte d'Ivoire. Afr. Sci., 13(3) 30-42.

DOI: http://www.afriquescience.info.

Kortatsi K, Tay K, Anomu G, Hayford E, Dartey A. 2007. Hydrogeochemical evaluation of groundwater in the lower Offin basin, Ghana; Environ Geol., p. 12.

Ligban R, Gone LD, Kamagate B, Saley MB, Biemi J, 2009. Processus hydrogéochimiques et origine des sources naturelles dans le degré carré de Daloa (Centre ouest de la Côte d'Ivoire). Int. J. Biol. Chem. Sci., 3(1): 38-47. DOI:

http://dx.doi.org/10.4314/ijbcs.v3i1.4273 3.

Mangoua MJ. 2013.Évaluation des potentialités et de la vulnérabilité des ressources en eau souterraine des 
aquifères fissures du bassin versant de la Baya (Est de la Côte d'Ivoire). Thèse de Doctorat. Université NanguiAbrogoua, Abidjan (Côte d'Ivoire). p. 171.

Nyamangara J, Jeke N, Rurinda J. 2013. Long term nitrate and phosphate loading river water in the Upper Manyame catchment, Zimbabwe. Water SA., 39(5): 637-642. DOI:

http://dx.doi.org/10.4314/wsa.v39i5.7.

OMS. 2011. Guidelines to Drinking-water Quality (fourth edition). (NLM classification: WA 675). OMS; p. 564.

Ouattara I, Kamagaté B, Dao A, Noufé D, Savané I. 2016. Processus de minéralisation des eaux souterraines et transfert de flux en milieu de socle fissuré: cas du bassin versant transfrontalier de la Comoé (Côte d'Ivoire, Burkina Faso, Ghana, Mali),
Int. J. Innov. Appl. Studies, 17(1): 57-69. DOI:

http://dx.doi.org/10.4314/jab.v102i1.5.

Shorieh GP, Razack M. 2015. Assessment of Groundwater Quality in the Dogger Aquifer of Poitiers, Poitou Charentes. $J$. Water Resour Prot., 7: 171-182. DOI: http://dx.doi.org/10.4236/jwarp.2015.730 14.

Youan ta M, Lasme OD, Baka D, Lasm T, Jourda PJ, Biemi J. 2015. Analyse des propriétés hydrodynamiques de l'aquifère fissuré du socle paléoprotérozoïque: Aide à l'approvisionnement en eau potable des populations de la région de Bondoukou (Nord-est de la Côte d'Ivoire). Int. J. Innov. Appl. Studies, 13(3): 561-580. DOI: http://www.ijias.issr-journals.org. 